\title{
CONTACTOS MEDIOTRUSIVOS EN LA OCLUSIÓN Y LA ATM
}

Mediotrusive contacts in occlusion and TMJ

Fecha de Recepción: 10 de febrero 2020
Contatos mediotrusivos em oclusão e ATM

Aceptado para su publicación: 31 de marzo 2020

\section{Autores: \\ Juan José Christiani ${ }^{1}$ Ricardo Altamirano ${ }^{2}$}

1. Área Disciplinar Preclínica de Prótesis. Módulo Introducción a la Odontología Rehabilitadora. Facultad de Odontología. Universidad Nacional del Nordeste. Argentina.

2. Área Disciplinar Oclusión. Módulo Introducción a la Odontología Rehabilitadora. Lugar de trabajo. Facultad de Odontología. Universidad Nacional del Nordeste. Argentina.

\section{Correspondencia:} Juan José Christiani

Correo electrónico: jjchristiani@hotmail.com jjchristiani@odn.unne.edu.ar

Conflicto de intereses: los autores declaran no tener conflictos de interés.

Fuente de financiamiento: autofinanciado.

\section{Resumen}

La oclusión ha sido definida como el contacto existente entre los dientes. Estos contactos pueden ser considerados tanto en estática, cuando los dientes contactan en máxima intercuspidación al finalizar el cierre mandibular, como en dinámica, es decir, cuando los dientes se deslizan entre sí con el movimiento mandibular. Los contactos mediotrusivos (contactos de no trabajo o de balance) son aquellos contactos oclusales que se encuentran entre las vertientes internas de las cúspides linguales maxilares y las internas de las cúspides bucales mandibulares del lado de no trabajo durante los movimientos de lateralidad. Estos contactos mediotrusivos podrían desencadenar trastornos temporomandibulares afectando la oclusión y la articulación temporomandibular. El objetivo de esta revisión fue analizar las características y la relación de los contactos mediotrusivos con la oclusión y la articulación temporomandibular. Los pacientes que presentan contactos mediotrusivos pueden generar en el tiempo, cambios en la función oclusal así como modificaciones en las trayectorias de los movimientos mandibulares que se traducen en alteraciones tanto a nivel de la oclusión como de la ATM. Por eso se considera importante la detección precoz de estos contactos para evitar alteraciones más graves.

Palabras clave: oclusión, ATM, contactos mediotrusivos.

\footnotetext{
Abstract

Occlusion has been defined as the contact between teeth. Those contacts could be considered both in static, when the teeth contact in maximum intercuspidation at the end of the mandibular closure, as in dynamic, that is, when the teeth slide with each other with
} 
the mandibular movement. The mediotrusive contacts (non-work or balance contacts) are those occlusal contacts that are located between the internal slopes of the maxillary lingual cusps and the internal ones of the mandibular buccal cusps of the non-work side during laterality movements, These mediotrusive contacts could trigger temporomandibular disorders affecting occlusion and the mandibular temporal joint. The aim of this review was to analyze the characteristics and relationship of mediotrusive contacts with occlusion and temporomandibular joint. Patients who have mediotrusive contacts can generate, after time, changes in occlusal function as well as changes in the trajectories of the mandibular movements that result in alterations both at the level of occlusion and ATM. Therefore, early detection of these contacts is considered important to avoid more serious alterations.

Key words: occlusion, ATM, mediotrusive contacts.

\section{Resumo}

A oclusão é definida como o contato entre os dentes. Esses contatos podem ser considerados tanto estáticos, quando os dentes entram em contato com máxima intercuspidação no final do fechamento mandibular, como dinâmicos, ou seja, quando os dentes deslizam entre si com o movimento mandibular. Os contatos mediotrusivos (contatos que não são de trabalho ou de equilíbrio) são aqueles contatos oclusais que estão localizados entre as inclinações internas das cúspides linguais superiores e as internas das cúspides vestibulares inferiores do lado não trabalho durante os movimentos de lateralidade. Esses contatos mediotrusivos podem desencadear desordens temporomandibulares que afetam a oclusão e a articulação temporal mandibular. O objetivo desta revisão foi analisar as características as caraterísticas dos contatos mediotrusivos e a sua relação com a oclusão e com a articulação temporomandibular. Pacientes que possuem contatos mediotrusivos podem gerar, com o tempo, alterações na função oclusal e alterações nas trajetórias dos movimentos mandibulares que resultam em alterações tanto no nível de oclusão quanto no ATM. Portanto, a detecção precoce desses contatos é considerada importante para evitar alterações mais graves.
Palavras-chave: oclusão, ATM, contatos mediotrusivos.

\section{Introducción}

Una de las funciones primordiales del sistema Estomatognático es la masticación. Para que dicha función se realice adecuadamente, es necesaria la interacción de condiciones musculares, la articulación temporomandibular, el contacto que se produce entre los dientes (oclusión) y el sistema neuromuscular ${ }^{1}$.

La oclusión ha sido definida como el contacto existente entre los dientes. Estos contactos pueden ser considerados tanto en estática, cuando los dientes contactan en máxima intercuspidación al finalizar el cierre mandibular, como en dinámica, es decir, cuando los dientes se deslizan entre sí con el movimiento mandibular².

La articulación temporomandibular (ATM) es aquella encargada de guiar los movimientos de la mandíbula. Durante los movimientos denominados excéntricos de la mandíbula (lateralidad y protrusión) pueden producirse varios tipos de patrones oclusales entre los dientes antagonistas, producto de la dinámica mandibular. El tipo de contactos que surge durante estos movimientos depende de varios factores como la relación entre los maxilares, la posición de los dientes, la morfología y la cantidad de desgaste dental, la función muscular, la cantidad de movimiento y la función de la ATM³ .

En odontología, la evaluación funcional tanto de la oclusión en estática y dinámica como de la ATM ha sido de gran importancia, por cuanto de ella dependen, en gran parte, el éxito o el fracaso de cualquier tratamiento. El establecer una oclusión ideal ha sido por mucho tiempo un requerimiento para realizar diagnósticos efectivos y/o evaluar los resultados de los distintos tipos de tratamientos realizados en los pacientes.

Algunos autores hacen una distinción terminológica, denominando oclusión a cada contacto estático entre uno o más dientes inferiores con uno o más dientes superiores, y refiriéndose como oclusión funcional a los contactos oclusales de los dien- 
tes maxilares y mandibulares durante la función, por ejemplo, durante la masticación y la deglución². Asimismo, existen múltiples conceptos para nominar los lados al realizar un movimiento mandibular lateral. En la literatura, el lado hacia el cual la mandíbula se mueve se llama laterotrusivo, de trabajo o de funcionamiento; mientras que el otro lado es llamado mediotrusivo, de no trabajo, balance o de no funcionamiento ${ }^{4}$.

La Academia de Prostodoncia (2005), sostiene que una interferencia en el lado de no trabajo es un contacto no deseado de las superficies oclusales. Es decir, los contactos mediotrusivos en movimientos de lateralidad pueden contribuir a la aparición de las alteraciones funcionales ${ }^{5,6}$.

Durante el movimiento mandibular lateral, los dientes posteriores mandibulares -derechos e izquierdos- se desplazan sobre los dientes antagonistas en distintas direcciones. Las posibles áreas de contacto se localizarán en diferentes zonas, y por lo tanto, recibirán distintos nombres ${ }^{5}$.

Los contactos mediotrusivos (contactos de no trabajo o de balance) son aquellos contactos oclusales que se encuentran entre las vertientes internas de las cúspides linguales maxilares y las internas de las cúspides bucales mandibulares del lado de no trabajo durante los movimientos de lateralidad. Cuando se realiza una lateralidad derecha, los dientes posteriores izquierdos mandibulares se desplazan en una dirección medial sobre los antagonistas. Las interferencias mediotrusivas, si existen, se encontrarán en el lado de no trabajo es decir, en el lado izquierdo en el movimiento lateral derecho y en el derecho en los movimientos de lateralidad izquierda. La presencia de estas interferencias determina que el movimiento sea crítico ya que se producirán tracciones a nivel de la cápsula con un alto potencial patogénico para la ATM ${ }^{7}$.

Como se puede apreciar, no está del todo definido el rol que juegan los distintos contactos mediotrusivos durante un movimiento mandibular y su influencia en el sistema estomatognático.

Okeson $^{17}$, señala que los contactos mediotrusivos son percibidos de manera diferente que otros tipos de contactos por el sistema nervioso central y debiesen ser evitados para brindar una oclusión funcional óptima. Hellmann et al. ${ }^{8}$, relacionaron los contactos mediotrusivos con dolor durante el movimiento mandibular y la sensibilidad muscular a la palpación, mientras que otros reportaron que los signos y síntomas de disfunción mandibular no eran dependientes de los contactos mediotrusivos ${ }^{10-12}$.

Las alteraciones o interferencias en la oclusión y la ATM pueden desencadenar los denominados trastornos temporomandibulares (TTM), que se caracterizan por presentar una serie de signos y síntomas, como dolor en el área bucofacial (de origen no dental, ni periodontal) y alteraciones funcionales, fundamentalmente las relacionadas con los ruidos articulares y las limitaciones a los movimientos mandibulares ${ }^{12}$.

Cada componente del Sistema Estomatognático, músculos, piezas dentarias, huesos, nervios y la ATM, posee un grado de tolerancia a la agresión, que si es superado, puede desencadenar un TTM ${ }^{15}$. La disfunción no se manifiesta en todas las personas por igual, sino que se refleja precisamente en esa estructura más débil que cada paciente tiene (menor tolerancia estructural) y que hace que la variabilidad sea aún más marcada entre unos individuos y otros.

En los últimos años, los trabajos que demuestran la nociva influencia de las interferencias en la articulación, han ampliado su campo de estudio al aspecto postural mostrando que determinados grupos musculares relacionados con la ATM también pueden verse afectados de manera evidente influyendo de manera indirecta en la articulación, manifestándose con dolor miofacial ${ }^{19-20}$. Cabe destacar que las interferencias en céntrica pueden coincidir con las de balanceo y ello tiene implicancia en el posterior tratamiento con un tallado selectivo ${ }^{16}$. Desde el punto de vista fisiopatológico, se produce un movimiento condilar anómalo con estiramiento y afectación de los tejidos blandos del lado de no trabajo y desviación de la mandíbula que puede traumatizar la articulación ${ }^{16}$. Los vectores de fuerza son modificados y el fulcro en el lado de trabajo que representaba el bolo alimenticio, se acompaña, ahora, por un segundo fulcro en el lado de balanceo, representado por 
la interferencia, (contactos mediotrusivos) lo que hace que el brazo de palanca sea más corto, alterando de esta manera, todo el sistema propioceptivo y neuromuscular del aparato estomatognático ${ }^{12-16}$.

Algunos estudios sugieren que los contactos mediotrusivos son percibidos por el sistema neuromuscular de manera distinta a los demás tipos de contactos oclusales; por lo tanto, se encargan de aumentar la actividad neuromuscular a través de la estimulación de los propioceptores y nociceptores del ligamento periodontal. De la misma forma, los contactos prematuros incrementan la contracción eléctrica de los músculos temporales en mayor proporción, comparado con los músculos masete$\operatorname{ros}^{14-16}$.

Para solucionar este problema, en el diagnóstico de la anomalía debe considerarse la posición de las bases esqueléticas en una correcta relación céntri$\mathrm{ca}$, teniendo en cuenta la influencia que sobre los dientes ejercen los músculos y ligamentos circundantes.

Esta temática es amplia y muchas veces contradictoria en ciertos aspectos, aunque la mayoría de los estudios o autores coinciden que existe una relación entre la presencia de una maloclusión y la aparición de la disfunción cráneo-temporomandibular (DTM) ${ }^{17}$.

Gran cantidad de individuos experimentan en algún momento de su vida signos o síntomas de TTM, cuya etiología es multicausal. Existen muchos criterios a la hora de determinar el grado de participación y comportamiento del factor oclusal en relación con los TTM.

Siempre que un análisis oclusal previo respalde la sospecha de que el estado oclusal constituye el factor etiológico primario como causa del TTM, y este pueda ser detectado, tratado y se alivie la sintomatología dolorosa, la terapia oclusal será el tratamiento eficaz para resolver este problema de salud.

Factores locales también pueden originar estos trastornos, como el cambio propioceptivo o sensitivo, una corona alta, con una oclusión inadecuada. También puede ser secundaria a un traumatismo, una inyección local y la apertura exagerada y prolongada que genera un esguince. El bruxismo, que es el golpeteo permanente o rechinar de los dientes en forma inconsciente en el sueño, es otro de los factores que puede desempeñar un papel importante en la génesis de los TTM.

Entre los factores sistémicos que pueden repercutir sobre el sistema masticatorio, se encuentra el stress emocional (que debe ser abordado por los especialistas), la genética, el sexo y, la dieta ${ }^{18,19}$.

Una de las causas que provocan las alteraciones de la ATM, son las interferencias del lado de balanceo, en las cúspides palatinas superiores de molares (vertientes vestibulares) y cúspides vestibulares de molares inferiores (vertientes linguales), donde se localizan los contactos mediotrusivos. Como se dijo anteriormente, estos contactos pueden afectar la oclusión y la ATM desencadenando un TTM.

Este tipo de contactos se deben examinar detenidamente. Para ello, se le pide al paciente que indique si nota interferencias al mover su mandíbula en mediotrusiva, luego se aplica fuerza firme en el ángulo goníaco en dirección supero medial pidiendo nuevamente al paciente que mueva en posición mediotrusiva. Con esto se contrarresta la protección neuromuscular y así se detectan los contactos mediotrusivos en forma asistida ${ }^{20}$.

El objetivo de esta revisión fue analizar las características y la relación de los contactos mediotrusivos con la oclusión y la articulación temporomandibular.

\section{Metodología}

Debido a que existe disparidad acerca del rol que cumplen los contactos mediotrusivos en los trastornos oclusales y su repercusión a nivel de la ATM surge la siguiente pregunta que motiva la presente revisión: ¿Qué relación existe entre los contactos mediotrusivos con alteraciones en la oclusión y la ATM?

Las bases de datos empleadas para la búsqueda fueron: Biblioteca Virtual de Salud (Red BVS), PubMed, Scielo durante el período 2009-2019. 
Se utilizaron como palabras claves de búsqueda los términos: contactos mediotrusivos, oclusión y trastornos temporomandibulares.

Como criterio de la búsqueda, se seleccionaron las siguientes variables como estrategia:

1) Variables demográficas: edad (expresado en años) y sexo (femenino o masculino).

2) Presencia de contactos mediotrusivos (contactos en el lado de NO trabajo identificados en movimientos de lateralidad, utilizando papel de articular)

3) Oclusión: Contacto de las piezas dentarias donde se evaluó la presencia de facetas de desgastes.

4) Estado de la articulación temporomandibular (ATM): Se analizó en base a las siguientes dimensiones o subvariables:

- En relación a los movimientos realizados en los tres planos del espacio (apertura mandibular, protrusión y lateralidad) .

- Presencia de ruidos articulares: determinada durante movimientos de apertura o de apertura y cierre mandibular.

- Presencia de dolor.

\section{Contactos mediotrusivos como desenca- denante de TTM}

El concepto de los contactos mediotrusivos en la función masticatoria es bastante reciente en odontología. La mandíbula funciona como una palanca de Clase III, una interferencia del lado de no trabajo producida por falta de desoclusión anterior, puede generar un cambio de palanca, ya que la interferencia del lado de no trabajo actuaría como apoyo, y la ATM pasaría a ser resistencia, pasando a un cambio de palanca de primer género ${ }^{7}$.

Si existen contactos posteriores mediotrusivos en los movimientos de lateralidad sin intervención del profesional se los considera como contactos mediotrusivos no ayudados. Por el contrario, si existen contactos posteriores mediotrusivos con intervención del profesional se los considera como contactos mediotrusivos ayudados ${ }^{21}$.

Los contactos mediotrusivos no ayudados, parecen afectar de manera negativa a la función masticatoria y constituyen, por lo tanto, un posible factor etiológico para una alteración funcional tanto den- taria como articular.

En una investigación sobre interferencias oclusales en pacientes con TTM, se observó que se producen más interferencias en el lado de no trabajo en movimientos de lateralidad ${ }^{22-24}$. En cambio, los contactos mediotrusivos que solo se presentan con fuerzas asistidas importantes, pueden proteger de hecho a la articulación homolateral durante una carga intensa, como sucede en el bruxismo.

Los TTM constituyen una patología característica de la población adulta que presenta en general desarmonías oclusales, entre ellas los contactos mediotrusivos que inciden de manera directa en la ATM y la oclusión.

Estas patologías se presentan con mayor frecuencia en el sexo femenino. Pareciera ser que las mujeres son más propensas a tener este tipo de alteraciones, debido a factores sociales como su estatus social, estados fisiológicos como los períodos menstruales, fatiga física y psíquica, menopausia, entre otros ${ }^{24}$.

Sharma ${ }^{18}$, afirma que la proporción entre mujeres y hombres es de por lo menos 4:1. Esto se puede explicar porque son ellas las que buscan tratamiento para los desórdenes de la ATM y además son quienes presentan los TTM más graves.

Luther ${ }^{25}$, encontró alta prevalencia de TTM en individuos de todo el mundo, variando, entre $7-84 \%$, con un rango de edad entre 18 y 74 años. Sin embargo, Manfredini et al. ${ }^{26}$, hallaron mayor frecuencia en personas en rangos de edad de 20 a 40 años, y menor prevalencia en niños y ancianos. Otros autores, afirman que los TTM tienden a iniciarse después de la pubertad, siendo que el aumento de la severidad de los signos y síntomas generalmente tiene su pico en la edad reproductiva, con mayores valores en edades comprendidas entre 20 y 40 años ${ }^{27}$.

El análisis oclusal en pacientes con contactos mediotrusivos debe ser una herramienta imprescindible en el examen clínico, ya que la literatura reporta que puede ser un factor desencadenante de TTM, los cuales se definen como alteraciones clíni- 
cas que comprometen los músculos de la masticación, la ATM y estructuras asociadas como ligamentos y disco articular. Su etiología es multifactorial e incluye factores biológicos, ambientales, sociales, emocionales y cognitivos, que por sí solos o asociados, pueden generar la aparición de TTM ${ }^{28}$.

Morquette et al. ${ }^{20}$, propusieron evaluar la función oclusal con actividad electomigráfica, tanto en pacientes con contactos mediotrusivos como sin ellos, y halló patrones similares en la actividad muscular entre ambos grupos.

\section{Interferencias en la oclusión}

Uno de los indicadores de alteración oclusal, es la presencia de facetas de desgastes. Kawagoe et al. ${ }^{29}$, afirman que existe una relación significativa entre facetas de desgastes de contactos mediotrusivos en la zona de los molares durante el bruxismo del sueño y la prevalencia de signos de disfunción temporomandibular.

Por el contrario, otras investigaciones le atribuyen posibles roles protectores de la articulación temporomandibular del mismo lado de los contactos mediotrusivos, en el lado de balance ${ }^{14-20}$.

Un coadyuvante de gran valor diagnóstico para el clínico, como sostiene Grau y $\mathrm{Cabo}^{30}$, es la restricción que se genera en los movimientos mandibulares, que constituye además un signo alarmante para los pacientes. En su estudio menciona interferencias oclusales, siendo en el lado de no trabajo las más frecuentes.

Por otro lado, Ruiseco Palomares et al. ${ }^{23}$, encontraron que la prevalencia de interferencias, durante los movimientos mandibulares asociadas a la alteración del plano oclusal en la población fue del $93 \%$. Afirman que las interferencias oclusales son el principal factor etiológico de las disfunciones de la ATM, donde el dolor muscular y articular son los síntomas de desorden temporomandibular más frecuente asociado a las interferencias y además, expresan que el mayor número de interferencias se presenta en los movimientos de protrusión $(46,2 \%)$ y de lateralidad en el lado de no trabajo.
Posiblemente, las interferencias en protrusiva podrían ser las más lesivas, pues pueden ocasionar una subluxación condilar del lado afectado, al pasar el punto de fulcro a la mandíbula. Las interferencias en balanza, ocasionan dolor miofacial heterópico y facetas de desgaste en las cúspides funcionales; además, el cambio del fulcro en esta interferencia ocasiona un brazo de palanca más corto alterando el sistema propioceptivo y neuromuscular, ocasionando un movimiento condilar incorrecto con estiramiento y afectación de los tejidos blandos del lado de no trabajo y desviación de la mandíbula que puede traumatizar la articulación ${ }^{26-29}$.

\section{Dolor y ruido característica clínica de TTM}

Chisnoiu et al..$^{28}$, evidenciaron que las interferencias en laterotrusión se relacionan principalmente con la presencia de ruido articular en un $57 \%$ e interferencia protrusiva en un $75 \%$. Por su parte, Rotemberg et al. ${ }^{31}$, observaron sólo un $13 \%$ de ruidos articulares en pacientes con TTM.

Valenzuela Zamora et al. ${ }^{32}$, en un estudio evaluaron el dolor teniendo en cuenta el mapa de Rocabado, refiriendo a la zona dos sinovial anterior como la de mayor frecuencia. Luego del uso de férulas oclusales a los 3 meses, reportaron la desaparición del dolor, con excepción de las zonas cinco sinovial posterior superior y seis sinovial posterior inferior.

Díaz Cevallos et al. ${ }^{33}$, afirman que la mayor frecuencia de dolor es por lesión del ligamento colateral, en pacientes que hayan recibido tratamiento de ortodoncia. Asimismo, Cabrera Villalobos et al. ${ }^{34}$, relaciona la presencia de dolor articular con interferencia oclusal y con factores de stress.

\section{Conclusiones}

Los pacientes que presentan contactos mediotrusivos pueden generar en el tiempo, cambios en la función oclusal así como modificaciones en las trayectorias de los movimientos mandibulares que se traducen en alteraciones tanto a nivel de la oclusión como de la ATM. Por eso, es importante la detección precoz de estos contactos para evitar alteraciones más graves. 


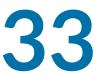

DIVULGACIÓN
Revista de la Facultad de Odontología

Contactos mediotrusivos en la Oclusión y la ATM
REFO

VOL. XIII | No 1| AÑO 2020

ISSN 1668-7280

ISSN-E 2683-7986
Las alteraciones en los movimientos mandibulares y la presencia de ruido son evidentes en pacientes que presentaban contactos mediotrusivos. Tanto la aparición de ruidos articulares, como las restricciones en los movimientos mandibulares y la presencia de dolor, son indicadores de alteraciones a nivel de la ATM.

Es importante tener en cuenta la variabilidad individual, ya que una interferencia mediotrusiva podría ser absolutamente incapaz de provocar algún desequilibrio en una persona y ser, sin embargo, muy dañina en otro paciente.

Ante la aparición de signos o síntomas de TTM, además de realizar un cuidadoso examen dental de rutina, es fundamental implementar un tratamiento oclusal, para restablecer su estabilidad. Es prioridad, conocer la biomecánica particular de cada paciente y su correlación con sus parámetros oclusales y biológicos.

Un proceso terapéutico estandarizado y particularizado, con especial observación de la presencia de contactos mediotrusivos, es la mejor vía para solventar de manera exitosa las disfunciones y evitar el elevado número de pacientes refractarios al tratamiento. El diagnóstico precoz, con un tratamiento con placas oclusales en pacientes jóvenes con interferencia mediotrusivas, estabilizará la oclusión y ATM para evitar mayores alteraciones en el futuro.

\section{Referencias Bibliográficas}

1. Chaithanya R, Sajjan S, Raju AVR. Un estudio del cambio en los contactos oclusales y la dinámica de la fuerza después del tratamiento protésico fijo y después del equilibrio - Uso de Tekscan III. J Indian Prosthodont Soc. 2019; 19 (1): 9-19. doi: 10.4103 / jips.jips_238_18

2. Chisnoiu AM, Picos AM, Popa S, Chisnoiu PD, Lascu L, Picos A, Chisnoiu R. Factores involucrados en la etiología de los trastornos temporomandibulares: una revisión de la literatura. Clujul Med. 2015; 88 (4): 473-8. doi: 10.15386 / cjmed-485

3. Tanaka EM, González MC, Díez I, López JP. Aplicación clínica del Bruxchecker® en odontología para la evaluación en sueño del patrón de desgaste oclusal. Univ Odontol. 2015;34(72):19-30. doi: 10.11144/Javeriana.uo3472.acbo

4. Johnson AW. Dental occlusion ties: A rapid, safe, and non-invasive maxillo-mandibular fixation technology. Laryngoscope Investig Otolaryngol. 2017;2(4):178-183. doi: 10.1002/lio2.77.

5. Julià-Sánchez S, Álvarez-Herms J, Cirer-Sastre R, Corbi F, Burtscher M. The Influence of Dental Occlusion on Dynamic Balance and Muscular Tone. Front Physiol. 2020;10:1626. doi: 10.3389/fphys.2019.01626

6. Leroux E, Leroux S, Maton F, Ravalec X, Sorel O. Influence of dental occlusion on the athletic performance of young elite rowers: a pilot study. Clinics (Sao Paulo). 2018;73:e453. doi: 10.6061/clinics/2017/e453

7. Hernández Reyes B, Díaz Gómez SM, Marín Fontela GM, Márquez Ventura Y, Rodríguez Ramos S, Lazo Nodarse R. Caracterización de la oclusión dentaria en pacientes con trastornos temporomandibulares. Arch méd Camaguey. 2018;22(5):708-725. Disponible en: https://www. redalyc.org/articulo.oa?id=211149710015

8. Hellmann D, Giannakopoulos NN, Schmitter M, Lenz J, Schindler HJ. Anterior and posterior neck muscle activation during a variety of biting tasks. Eur J Oral Sci. 2012;120(4):326-34. doi: 10.1111/j.16000722.2012.00969.x

9. Augusto VG, Perina KCB, Penha DSG, Dos Santos DCA, Oliveira VAS. Temporomandibular dysfunction, stress and common mental disorder in university students. Acta Ortop Bras. 2016;24(6):330-333. doi: 10.1590/1413785220162406162873

10. Gil-Martínez A, Paris-Alemany A, López-de-Uralde-Villanueva I, La Touche R. Manejo del dolor en pacientes con trastorno temporomandibular (TMD): desafíos y soluciones. J Pain Res. 2018;11:571-587. doi: 10.2147 / JPR.S127950 


\section{REFO}

VOL. XIII | N 1| AÑO 2020

ISSN 1668-7280

ISSN-E 2683-7986
11. Camacho AD, Velásquez Cujar SA. Aceleración del movimiento dental: revisión de la literatura por un método alternativo de evidencia científica. World J Methodol. 2014;4(3):151-62. doi: 10.5662 / wjm.v4.i3.151

12. Altamirano R, Collante C, Christiani J. Estudio Descriptivo de transtornos temporomandibulares en estudiantes universitarios. Revista de la Facultad de Odontología UNNE. 2018; XI(1):16-21. Disponible en: file://D:/ Cliente/Downloads/3861-12320-1-PB.pdf

13. Zuluaga López O. Evaluación funcional de las restauraciones protésicas fijas. Reporte de serie de casos. Univ Odontol. 2017;35(75):1-13. doi: 10.11144/Javeriana. uo35-75.efrt

14. Bhargava D, Jain M, Deshpande A, Singh A, Jaiswal J. Temporomandibular joint arthrocentesis for internal derangement with displacement without reduction. J Maxillofac Oral Surg. 2015;14(2):454-9. doi: 10.1007/ s12663-012-0447-6

15. Cobos Castro I, Gutiérrez Segura M, Montero Sera E, Zamora Guevara N. Trastornos temporomandibulares en pacientes bruxópatas, trabajadores de estomatología de Mayarí. CCH, Correo cient. Holguín. 2017;21(3):734-747. 16. Silva Contreras, A. M. Bruxismo: su comportamiento en un área de salud. Rev. Ciencias Médicas. 2015;19(1):5665. Disponible en: http://www.revcmpinar.sld.cu/index. php/publicaciones/article/view/1987/html_53

17. Okeson JP. Management of Temporomandibular Disorders and Occlusion. 7th ed. Mosby, Elsevier: St. Louis, MO, USA;2013.

18. Sharma S, Gupta DS, Pal US, Jurel SK. Etiological factors of temporomandibular joint disorders. Natl J Maxillofac Surg. 2011;2(2):116-9. doi: 10.4103/09755950.94463

19. Nuño Heredia KV, Popoca Hernández EA, Carrillo Arellano J, Espinosa de Santillana I, Martínez Martínez RE. Tipo de bruxismo en pacientes con trastornos temporomandibulares de acuerdo al sexo. Revista Mexicana de Estomatología. 2019;6(1):26-32. Disponible en: https:// www.remexesto.com/index.php/remexesto/article/ view/256/483

20. Morquette P, Lavoie R, Fhima MD, Lamoureux X, Verdier D, Kolta A. Generation of the masticatory central pattern and its modulation by sensory feedback. Prog Neurobiol. 2012;96(3):340-55. doi: 10.1016/j.pneurobio.2012.01.011

21. Dos Santos RB, Queiroz AB, Fidelis RJR, Lopes CF, de Araújo JS Filho. Subluxação da mandíbula para abordagem de bifurcação carotídea alta em paciente com parotidite by contraste iodado: relato de caso y revisión de literatu- ra. J Vasc Bras. 2017;16(4):329-334. doi: 10.1590/16775449.001117

22. Soto L, De la Torre J, Aguirre I, De la Torre E. Trastornos Temporomandibulares en pacientes con maloclusiones. Revista Cubana de Estomatología. 2013; 50(4):37487. Disponible en: http://scielo.sld.cu/pdf/est/v50n4/ est05413.pdf

23. Ruiseco Palomares A, Llanes Rodríguez M, Rodríguez Calzadilla OL, Rodriguez Aparicio A. El dolor articular y su relación con las interferencias oclusales. Rev Habanera Ciencias Médicas. 2014;13(3):417-24. Disponible en: https://www.medigraphic.com/pdfs/revhabciemed/hcm2014/hcm143g.pdf

24. Garrigós-Pedrón M, Elizagaray-García I, Domínguez-Gordillo AA, Del-Castillo-Pardo-de-Vera JL, Gil-Martínez A. Temporomandibular disorders: improving outcomes using a multidisciplinary approach. J Multidiscip Healthc. 2019;12(3):733-747. doi: 10.2147/JMDH. S178507

25. Luther F. TMD and occlusion part I, damned if we do? Occlusion: The interface of dentistry and orthodontics. $\mathrm{Br}$ Dent J. 2007;202(1):1-7. doi:10.1038/bdj.2006.122

26. Manfredini D, Perinetti G, Stellini E, Di Leonardo B, Guarda-Nardini L. Prevalencia de las características de maloclusión dental estática y dinámica en dos subgrupos de pacientes con trastornos temporomandibulares: Implicaciones de la epidemiología de la asociación entre TTM y oclusión. Quintessence Int. 2015;3(8):600-609.

27. Santana M, Moreno Y, Rosales K, Osorio Y, Morales L. Grado de Disfunción temporomandibular en pacientes mayores de 19 años. MEDISAN. 2013; 17(12):90879093. Disponible en: http://scielo.sld.cu/pdf/san/ v17n12/san091712.pdf

28. Chisnoiu AM, Buduru S, Lascu LM, Vesa SC, Picoş $A$, Pascu L, et al. Influence of occlusal characteristics on temporomandibular joint disorder development - a cross-sectional study. Hum Vet Med. 2015;7(3):197201. Disponible en: http://www.hvm.bioflux.com.ro/ docs/2015.197-201.pdf

29. Kawagoe T, Onodera K, Tokiwa O, Sasaguri O, Akimoto S, Sato S. Relationship between sleeping occlusal contact patterns and temporomandibular disorders in the adult Japanese population. Int J Stomatol Occlusion Med. 2009;2:11-5. Disponible en: https://doi.org/10.1007/ s12548-009-0002-3

30. Grau León I, Cabo G. Evaluación de la oclusión en pacientes con trastornos temporomandibulares y desarmonías oclusales. Rev Cubana Estomatol. 2010; 47(2): 169-177. Disponible en: http://scielo.sld.cu/scielo.php?s- 
cript=sci_arttext\&pid=S003475072010000200005\&ln$\mathrm{g}=\mathrm{es}$.

31. Rotemberg E, Sanguinetti M, Massa F, Triaca J, Kreiner M. Prevalencia de signos y síntomas de trastornos temporomandibulares en una población joven al inicio del tratamiento por drogodependencia. Odontoestomatología. 2018;20(31):44-52. Disponible en: http://dx.doi. org/10.22592/ode2018n31a5

32. Valenzuela-Zamora YH, Moreno-Terrazas E, García-Aispuro FJ, Garnica-Palazuelos JC, Villalobos Rodelo JJ, García-Jau RA. Evaluación de los Cambios sintomatológicos a través del mapa del dolor después del uso de una férula oclusal para desprogramar la articulación temporo-mandibular. Rev Med UAS. 2017; 7(2):66-73. Dis- ponible en: http://hospital.uas.edu.mx/revmeduas/pdf/ v7/n2/mapadeldolor.pdf

33. Díaz Cevallos A, Gustavo Vinueza N, Paredes Jara C. y col. Mapeo del dolor de la articulación temporomandibular en adolescentes que hayan recibido tratamiento de ortodoncia. Rev cuban invest bioméd. 2018;37(4):1-7. Disponible en:http://scielo.sld.cu/scielo.php?script=sci_ arttext\&pid=S086403002018000400014\&lng=es.

34. Cabrera Villalobos Y, Álvarez Llanes M, Gómez Mariño M, Malcom Castillo ME. Oclusión y estrés en el síndrome dolor-disfunción temporomandibular: presentación de un paciente. AMC. 2009; 13(3). Disponible en: http://www. revibiomedica.sld.cu/index.php/ibi/article/view/204/ html 